\title{
A systems biology approach to understand the effects of diet on lifespan
}

Jin, K et al. PLoS Genetics https://doi.org/10.1371/journal.pgen.1008835 (2020)

Dietary restriction (DR), which refers to a reduction in food intake without malnutrition, effectively prolongs lifespan in various species. However, several studies in rodents and Drosophila have shown that this effect is not universal and DR response can vary between strains and genotypes. To identify the mechanisms underlying this variation, a team of investigators led by Daniel Promislow from University of Washington exposed 178 inbred Drosophila melanogaster lines to either a DR or ad libitum (AL) diet. By comparing lifespan response to DR in the different strains and integrating genomic and metabolomic data, the team was able to identify several gene-metabolite networks involved in DR response variation.

"We need to understand not only the mechanisms underlying DR itself, but also the mechanisms that influence variation in the DR response within a population," explain the investigators in their report. To do so, they used 178 inbred fly lines from the Drosophila Genetic Reference Panel (DGRP), a collection of fully sequenced inbred strains derived from a single wild population commonly used for genome-wide association analysis of phenotypic traits. Flies from each strain were placed into vials of either high (AL) or low (DR) yeast extract food, and fly deaths were recorded over time to compare lifespan for each strain ${ }^{\star}$ diet combination. Samples were also frozen 5 days after beginning the experimental diet for metabolic profiling. "This is the largest Drosophila study we know of to leverage both the metabolome and genome of a large number of genotypes of the same species to study lifespan response to DR," write the investigators.

The results show that the lifespan response to DR varied widely between strains as $70.8 \%$ of the lines lived longer, $18.6 \%$ did not show any change in lifespan and $10.5 \%$ lines had shorter lifespan
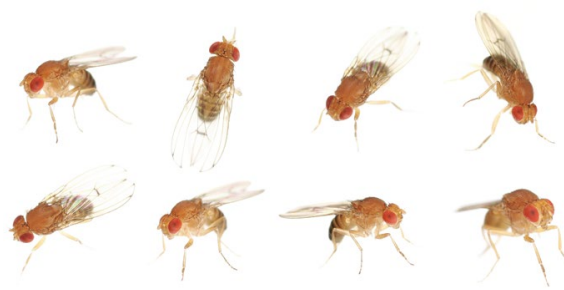

Drosophila melanogaster. Credit: Antagain/E+/ Getty

compared to flies fed AL. Among the 105 metabolites measured, 24 metabolites, including $\alpha-\mathrm{KG}$ and glutamine, were associated with the relative change in lifespan (rLS), a measure of the absolute different between DR and AL lifespan. The investigators also measured the covariance among metabolites and performed a differential network analysis to investigate the interactions between metabolites across diets. Analysis of these diet-dependent changes enabled the identification of 'hubs' in the metabolite networks, such as arginine and threonine, which gained numerous positive correlations under AL and DR diet respectively.

To confirm the role of the $\alpha-\mathrm{KG} /$ glutamine pathway in the effect of diet on lifespan, the investigators used RNA interference (RNAi) to knock down the expression of glutamate dehydrogenase (GDH), which catalyzes the reversible conversion of $\alpha \mathrm{KG}$ to glutamate, and glutamine synthetase 1/2 (GS1, GS2), which is responsible for the synthesis of glutamine. Analysis of the lifespan of the RNAi fly strains exposed to either to AL and DR diets revealed that whole-body and neuronal specific knockdown of $g s 2$ increased lifespan of AL flies, whereas lifespan of DR flies was not affected. Knockdown of $g d h$ and $g s l$ affected lifespan to a lesser extent.
These findings suggest that GS2 is part of a neuron-specific pathway that influences lifespan under AL.

Next, the investigators used a metabolic and genetic bipartite network analysis to search for genes that influence lifespan response to DR and identified $\mathrm{CCH} a 2 \mathrm{R}$, a gene that encodes a neuropeptide receptor involved in nutrient sensing and satiety. Knocking down $\mathrm{CCHa} 2 \mathrm{R}$ using a whole-body or a neuronal specific driver (GAL4-GeneSwitch (GAL4-gs) drivers) increased lifespan under DR, but had almost no effect on lifespan under AL. "To our knowledge, this is the first study to implicate CCHa2R in an aging/DR-related signaling mechanism. We hypothesize that CCHa2R is associated with lifespan due to its influence on metabolism," explain the investigators in their discussion. Bombesin receptor subtype 3 , the mammalian homolog of $\mathrm{CCHa} 2-\mathrm{R}$ shares a similar pattern of expression and has been implicated in the regulation of glucose metabolism, which suggests that the genes and pathways governing diet-specific lifespans are conserved.

"Taken together, these results support our network analysis as a map of gene-to-metabolite-to-phenotype pathways that underlie natural variation in the DR response and also highlight the fact that DR response can be influenced through a change in AL lifespan, DR lifespan, or both," write the investigators. They also stress that it is critical to explore the mechanisms of aging not only in single laboratory strains, but also in the context of genetically heterogeneous animal models, notably to better predict individual responses to lifespan-extending interventions.

Alexandra Le Bras

Published online: 17 August 2020 https://doi.org/10.1038/s41684-020-0632-z 